\title{
L'écosystème industriel
}

\section{Difficulté épistémologique d'une telle analogie}

\author{
Gérald Hess \\ Philosophe, Université de Lausanne, Institut des politiques territoriales et de l'environnement humain (IPTEH), 1015 Dorigny, \\ Suisse
}

\author{
Mots-clés : \\ écologie industrielle; \\ modèle ; \\ métaphore ; \\ épistémologie ; \\ développement \\ durable
}

\section{Keywords:}

industrial ecology;

model;

metaphor;

epistemology;

sustainable

development
Résumé - L'écologie industrielle propose une manière originale d'aborder les activités économiques. Elle se fonde pour cela sur l'analogie établie entre certains objets étudiés par l'écologie scientifique (l'écosystème, le métabolisme, la symbiose, la biocénose, etc.) et le système industriel. Toutefois, cette relation d'analogie soulève des difficultés en raison des interprétations différentes qu'elle suggère. Si l'on s'accorde sur sa fonction heuristique, l'analogie peut néanmoins se comprendre soit comme un modèle, soit comme une métaphore. Le présent article s'efforce d'abord de montrer en quoi le modèle diverge de la métaphore. Il cherche ensuite à justifier la pertinence épistémologique de cette distinction pour la recherche au sein de l'écologie industrielle. La réflexion devrait ainsi contribuer à clarifier le débat sur le rôle (supposé ou souhaité) de l'analogie dans le domaine de l'écologie industrielle et renforcer l'intérêt que présente ce champ d'investigations pour la mise en œuvre du développement durable.

\begin{abstract}
Industrial Ecosystem. Epistemological Challenge of such Analogy. Industrial ecology offers an original way of looking at economic activities. The approach is based on an analogy between certain objects studied by the science of ecology (ecosystems, metabolisms, symbiosis, biocenosis, etc.) and industrial systems. However, this analogical relationship raises difficulties due to the various interpretations it is open to. Although there is agreement regarding its heuristic function, the analogy can nevertheless be understood either as a model or a metaphor. The present article first attempts to show how models differ from metaphors. It then sets out to justify the epistemological relevance of this distinction for industrial ecology research. It endeavours describing how confusion amongst metaphors and models misleads to error. This consists in mixing up two levels of discourses, the first one refers to the reality and the second one reflects the vision created by the first one. The mistake may appear as an ideology or may damage the coherence of the approach of the industrial ecology. The reflection should thus contribute to clarifying the debate on the (supposed or desired) role of analogy in the field of industrial ecology and heighten the interest this field of investigation represents for implementing sustainable development.
\end{abstract}

L'écologie industrielle se profile depuis une vingtaine d'années comme une stratégie opératoire du développement durable. Il n'y a pas de définition standard de cette approche. Néanmoins, relève Suren Erkman (2004), l'un des spécialistes dans ce domaine, plusieurs traits distinguent l'écologie industrielle d'autres façons de représenter l'activité industrielle et économique. Résumons ces caractéristiques. L'écologie industrielle propose une vision globale, intégrée, de tous les composants du système industriel et de leurs relations avec la biosphère. En prenant appui sur les dynamiques technologiques,

Auteur correspondant : gerald-ernest.hess@unil.ch cette stratégie s'efforce d'optimiser les flux de matière et d'énergie du système industriel selon le fonctionnement des écosystèmes naturels.

Une terminologie inédite résulte de cet oxymore. Le «métabolisme industriel» désigne, par exemple, la mesure des flux de matière et d'énergie résultant des activités industrielles; la «biocénose industrielle » renvoie, quant à elle, à l'idée d'associer certains agents économiques afin d'optimiser les flux de matière et d'énergie de leurs activités ; enfin, la «symbiose de Kalundborg » est devenue l'expression consacrée pour désigner cette ville du Danemark où certains déchets issus d'entreprises ont été systématiquement transformés en ressources, contribuant 
ainsi à la production de biens d'autres entreprises situées sur le même territoire ${ }^{1}$.

Sur le plan épistémologique, on est néanmoins en droit de penser que l'analogie entre écosystème naturel et activités industrielles - sur laquelle se fonde l'écologie industrielle - reste encore trop vague pour être totalement opératoire. Car cette relation peut s'interpréter de plusieurs manières. Comment comprendre, en effet, l'usage de la notion d'écosystème naturel (et d'autres concepts issus de l'écologie) au sein de l'écologie industrielle? Ne s'agit-il vraiment que d'un usage ordinaire? Et ce dernier est-il bien de l'ordre de l'emprunt au domaine traditionnel de l'écologie scientifique? Dans ce cas, nous serions alors face à une simple extension du domaine d'application du concept d'écosystème à un autre domaine : le système industriel. Et l'analogie relèverait du modèle.

Or, il se pourrait, au contraire, que l'usage de la notion d'écosystème soit davantage qu'un emprunt à l'écologie classique. Il signerait plutôt l'émergence d'un sens inédit qui s'exprimerait par la métaphore inventive. Lorsque les chercheurs en écologie industrielle prennent la peine de réfléchir à cette relation d'analogie, ils font souvent l'amalgame entre métaphore et modèle. Ainsi en est-il de Ralf Isenmann (2003). Et ceux qui font la distinction n'ont pas pleinement conscience des implications. C'est ce qui ressort, par exemple, des contributions de John Ehrenfeld (2003 et 2007). Il me semble, par ailleurs, que le vif débat qui oppose les tenants de l'écologie industrielle sur la question du rôle (supposé ou souhaité) de la métaphore - Christoph Bey (2001), Jouni Korhonen (2005) et Peter E. Wells (2006) en fournissent une bonne illustration gagnerait beaucoup en clarté si l'on différenciait le modèle de la métaphore.

Je souhaite examiner cette ambiguïté qui n'est pas sans conséquence. À défaut d'une élucidation philosophique, l'équivoque est susceptible de conduire à une méprise épistémologique et ce, à l'insu de ceux qui se fondent sur cette analogie. L'erreur consiste, selon moi, à confondre ce qui relève finalement des propriétés objectives d'un état de choses avec la représentation de celui-ci. Autrement dit, elle consiste à mélanger deux niveaux de discours, le premier référant au réel, le second renvoyant à la vision qu'on s'en fait. Cette erreur peut se décliner sur plusieurs modes. Elle peut, par exemple, prendre la forme de l'idéologie. Ou bien, sans aller jusque-là, elle peut éventuellement porter préjudice à la cohérence et à l'originalité de l'approche de l'écologie industrielle.

Mais, avant d'aborder de front ce problème, un bref détour par les notions de métaphore et de modèle s'impose.

\footnotetext{
${ }^{1}$ Le site de Kalundborg est le cas le plus connu. Mais il en existe beaucoup d'autres, tels ceux de Kwinana en Australie, de Guitang en Chine ou encore le Fairfield Ecological Industrial Park à Baltimore, aux États-Unis.
}

\section{Métaphore et modèle}

La distinction entre métaphore et modèle requiert avant toute chose de savoir de quoi l'on parle. Par l'expression " écologie industrielle », je signifie, conformément à l'usage, le domaine d'étude portant sur certains « objets » : un système industriel, les flux de substances d'une activité économique, ses déchets, des micropolluants, etc. Dans le langage articulé, l'expression "écologie industrielle » est, comme le souligne S. Erkman, un oxymore. Il s'ensuit que des expressions comme "écosystème industriel ", «symbiose de Kalundborg » ou «biocénose industrielle» désignent les objets d'étude propres à l'écologie industrielle. Sur le plan linguistique, je les définis comme des métaphores ou des modèles ${ }^{2}$.

$C^{\prime}$ est au philosophe américain Max Black que l'on doit d'avoir, parmi les précurseurs, tenté un rapprochement entre le modèle et la métaphore ${ }^{3}$. Ses analyses font partie des références incontournables et bien connues en la matière ( $c f$. Black, 1962 et 1979). Traduire une métaphore par une paraphrase, soutient-il, laisse échapper quelque chose d'irremplaçable qu'on ne peut exprimer différemment ${ }^{4}$. C'est ce qui arrive, par exemple, lorsque l'on substitue, dans la phrase «L'amour est un rasoir $^{5}$ », le terme « rasoir»

\footnotetext{
2 Notons que l'expression "économie circulaire », que d'aucuns souhaitent substituer à "écologie industrielle», ne change rien à la présente problématique. Le présupposé, dans les deux cas, consiste bien à s'inspirer du fonctionnement quasi cyclique des écosystèmes biologiques pour décrire et comprendre les activités économiques et industrielles dans l'optique de la durabilité.

${ }^{3}$ Il existe d'innombrables travaux sur la métaphore tout comme sur le modèle. Récemment, Franck Varenne (2006) a montré que le rapprochement entre modèle et métaphore relève d'une posture qu'il qualifie de « linguisticiste » : on recourt à une figure du langage pour penser le modèle. Cela n'est, bien sûr, pas innocent. En effet, on présuppose ainsi une réalité accessible uniquement à travers le langage (via le concept de référence). Or, ajoute-t-il, les modèles de simulation computationnelle ne se laissent plus penser dans ce cadre étroit. En ce sens, je suggère qu'on pourrait peut-être déceler des similarités entre ce dernier type de modèle et ma propre conception de la métaphore ( $c f$. Hess, 2004). Néanmoins, je ne souhaite pas engager ici un débat épistémologique sur le modèle. Mon propos consiste plutôt à me servir des travaux de M. Black afin d'illustrer sur l'exemple de l'écologie industrielle les effets fâcheux d'une confusion entre modèle et métaphore. Ces deux notions sont donc employées, ni plus ni moins, comme des outils pour identifier certains problèmes épistémologiques de l'écologie industrielle.

4 Précisons que Black (1979) distingue les métaphores «actives » des métaphores «dormantes ». Contrairement aux secondes, les premières sont investies d'une nouvelle signification. La suite de notre réflexion se limitera aux métaphores actives, que certains auteurs, parmi beaucoup d'autres, nomment aussi les métaphores « vives » (Ricœur, 1975), "natives » (Nouvel, 2000) ou encore «nouvelles » (Lakoff et Johnson, 1980).

${ }^{5}$ J'emprunte cet exemple à Lakoff et Johnson (1980). Il illustre parfaitement l'aspect déconcertant suscité par les métaphores
} nouvelles. 
par « danger » ou «blessure possible». La métaphore permet à première vue de dire quelque chose à propos d'autre chose. Mais, de surcroît, le sens exprimé à travers elle est inédit, nouveau et, par conséquent, singulier.

C'est précisément ce double aspect - référentiel et créatif/innovant - de la métaphore qui incite $\mathrm{M}$. Black à rapprocher la métaphore du modèle.

\section{La spécificité du modèle}

Notons toutefois que ce rapprochement ne suffit pas à identifier la structure d'un modèle à celle d'une métaphore. Car le modèle présente des caractéristiques qui lui sont propres. Tout d'abord, il est une construction qui vise, à la manière d'un filtre, à simplifier les phénomènes, à les organiser, à sélectionner certains aspects au détriment d'autres. C'est ainsi que la double hélice des généticiens James Watson et Francis Crick permet de représenter la structure de la molécule d'ADN.

Ensuite, et c'est un point important, le sens ordinaire ou littéral du modèle conduit à élaborer un nouveau système d'implications de l'état de choses en question. La juxtaposition du domaine de référence avec la représentation qui sert de modèle engendre une autre façon de voir la réalité. Elle produit un isomorphisme entre la chose et le modèle qui la décrit. Par exemple, la modélisation de la molécule d'ADN sous la forme hélicoïdale de la double hélice permet de mettre en évidence une propriété nouvelle de cette substance : celle-ci conserve et transmet une information à la descendance (Nouvel, 2000) ${ }^{6}$. Le modèle de la double hélice réorganise donc le champ des implications relatives à la substance chromosomique de la cellule en fournissant des descriptions inédites des propriétés moléculaires.

Voilà pourquoi le modèle possède une dimension cognitive, qui constitue son troisième trait. Non seulement il décrit le réel, mais, surtout, il le re-décrit. La double hélice est bel et bien une redescription de la structure moléculaire telle qu'elle était perçue avant la percée de Watson et Crick.

\section{La spécificité de la métaphore}

Sur tous ces points, la métaphore diverge du modèle. Comme le suggère le philosophe des sciences Pascal Nouvel (ibid.), la métaphore définit en premier lieu le surgissement d'une complication sémantique dans le discours, un phénomène qui a fait l'objet d'une analyse approfondie par le philosophe Paul Ricœur (1975). Je me

\footnotetext{
${ }^{6}$ Nouvel précise que la double hélice avec appariement des bases de Crick et Watson est une représentation simplifiée - un modèle - permettant de comprendre les règles de Chargaff selon lesquelles il y a, dans les molécules d'ADN d'espèces différentes, parité des quantités de cytosine et de guanine, ainsi que des quantités d'adénine et de thymine.
}

contenterai ici de rappeler ce qu'Aristote (1991) remarquait déjà au moment où une métaphore est formulée, à savoir les effets d'étrangeté et de surprise suscités par l'incongruité sémantique.

En deuxième lieu, et c'est la conséquence de ce qui précède, la métaphore ne renvoie pas, selon moi, à un quelconque état de choses mais à une perspective ${ }^{7}$. Dans la phrase "L'amour est un rasoir », le rasoir n'est pas, en dépit des apparences, une nouvelle propriété objective de cette réalité qu'est l'amour. La métaphore opacifie, au contraire, le rapport entre le langage et l'objet auquel il renvoie en y introduisant un point de vue, une perspective subjective.

En dernier lieu, et corollairement, la figure métaphorique porte donc moins sur les choses qu'elle ne constitue une représentation des choses. En l'absence d'une référence propre, la métaphore - à condition qu'elle soit « active » ou « vive » - n'a pas de contenu cognitif spécifique. En d'autres termes, elle n'exprime pas la relation d'un locuteur au réel, mais le rapport d'un locuteur avec ses propres représentations du réel.

\section{L'écosystème naturel comme modèle du système industriel}

Les brèves distinctions épistémologiques qui précèdent permettent maintenant de comprendre les diverses implications de certains concepts ${ }^{8}$ propres à l'écologie scientifique pour décrire les activités industrielles. Considérons le premier cas de figure : quelle signification donner au fait d'envisager l'écosystème biologique comme un modèle?

L'écosystème biologique est un concept complexe. Il renvoie à de nombreuses notions, comme celles de niche écologique, de producteur (autotrophe), de consommateur (hétérotrophe), de réseau trophique, de décomposeurs, de flux de matière comme le carbone ou l'azote, de cycle, de chaîne alimentaire, de biocénose et de symbiose, etc. C'est pourquoi, perçue comme un modèle, la notion d'écosystème entraîne, pour commencer, une délimitation

7 J'ai eu l'occasion d'exposer mon point de vue à ce sujet - divergeant de celui de Black - dans une publication antérieure (cf. Hess, 2004). Certes, je ne conteste pas le fait que les sciences usent de métaphores. Mais soit elles le font avec une visée pédagogique, et alors les métaphores ne sont pas nouvelles, soit l'usage de métaphores est heuristique. Dans ce cas, il me semble important et utile de distinguer la métaphore du modèle : la métaphore - contrairement au modèle - ne présente pas, selon moi, une relation référentielle à proprement parler ( $c f$. Hess, 2003).

8 Nous nous restreignons dans ce qui suit au concept d'écosystème biologique, sans préjuger de l'intérêt qu'il y a, éventuellement, à élargir l'analogie à d'autres concepts de l'écologie scientifique, comme le suggère Peter E. Wells (Wells, 2006 ; Wells et Darby, 2006). 
des représentations implicites susceptibles de servir à organiser le système industriel. Une délimitation de cette sorte se produit, par exemple, avec l'idée de chaîne alimentaire, qui renvoie au fait que les déchets des uns sont des ressources pour les autres.

Ce réseau d'implications relatif au concept d'écosystème biologique est ensuite projeté sur le domaine constitué par le système industriel. Cette projection va induire une autre façon de voir l'activité industrielle. Précisément, elle permet de construire un «nouveau » réseau de représentations implicite au système industriel, en ce sens que ce réseau dévie intentionnellement des représentations traditionnelles associées au fonctionnement industriel et économique. Dans ce but, elle se sert de représentations comme celles de circularité, de chaîne alimentaire, relatives à l'écosystème naturel, afin de changer la conception d'une activité industrielle qui ne tient pas compte de la biosphère et de ses limites. Ainsi, il ne s'agira plus de stocker des déchets mais de les valoriser autant que possible comme matière première à consommer pour $\mathrm{d}^{\prime}$ autres activités ${ }^{9}$.

On relèvera, pour finir, un aspect important de cette projection caractéristique du modèle. Le concept d'écosystème comme modèle pour l'activité industrielle est envisagé dans son sens initial, c'est-à-dire tel qu'on l'entend au sein de l'écologie scientifique. C'est précisément en ce sens-là qu'il peut être plus ou moins fécond pour réorganiser le système industriel de manière inédite.

Deux conséquences de l'interprétation ci-dessus méritent d'être signalées. La première consiste en une extension du domaine d'objets de l'écologie scientifique. Par exemple, certaines propriétés d'une symbiose - pensons à la réciprocité, la constance, l'équilibre, l'interdépendance - qualifient également les liens entre les acteurs d'un système industriel réel. Bien entendu, toutes les caractéristiques des objets de l'écologie ne se laissent pas transposer simplement aux activités industrielles ${ }^{10}$. La régularité, l'abondance, la diversité ne sont pas forcément des propriétés des symbioses industrielles - tout

\footnotetext{
${ }^{9}$ Sur le site de Kalundborg, par exemple, l'eau usée de la raffinerie Statoil sert à refroidir la centrale électrique Asnaesvaerket. Celle-ci, à son tour, produit non seulement de l'électricité mais aussi de la vapeur d'eau, revendue à la raffinerie et à d'autres consommateurs de $l^{\prime}$ « écosystème ».

10 Stephen H. Levine (2003) a exploré certaines des limites de l'écosystème biologique comme modèle pour le système industriel. Par exemple, le système industriel se focalise essentiellement sur la demande d'un produit (l'output) qui détermine les ressources nécessaires pour le produire. L'écosystème naturel, en revanche, s'oriente vers les ressources (l'input) et le produit est déterminé par elles (la survie du lion dépend des proies à disposition). Il faut toutefois distinguer entre ce qui n'est pas analogue et ne peut l'être (exemple ci-dessus) et ce qui n'est pas analogue actuellement mais pourrait l'être (la production de déchets non valorisés).
}

comme l'entreprise, qui n'est pas à proprement parler un organisme vivant.

La seconde conséquence réside dans le fait que le modèle possède toujours une référence. Il doit y avoir une réalité à laquelle le modèle s'applique et qui permet de la décrire autrement qu'elle ne l'est traditionnellement. Dans la pratique, la démarche de l'écologie industrielle se traduit dans un premier temps par des analyses du métabolisme industriel ou des symbioses industrielles. Il s'agit alors d'effectuer des bilans de masse et d'énergie. Dans un second temps, celui de l'action, on développe des stratégies d'efficience, comme la décarbonisation ou la dématérialisation des produits, par exemple ${ }^{11}$.

\section{L'écosystème biologique comme métaphore du système industriel}

On envisagera l'écosystème biologique comme une métaphore du système industriel ou économique en s'inspirant des travaux du philosophe Ludwig Wittgenstein : voir quelque chose comme autre chose ( $c f$. Wittgenstein, 1953; Black, 1979; Ricœur, 1975). Ainsi, voir l'activité industrielle comme un écosystème, c'est la penser d'une certaine manière, sur l'arrière-fond de sa conception standard, c'est la concevoir ainsi plutôt que sur un mode traditionnel. Pour illustrer ce point, Wittgenstein reprend la célèbre figure " canard-lapin » du psychologue américain Joseph Jastrow : l'image dessinée d'un canard peut être perçue soudainement comme celle d'un lapin et inversement. Cet exemple montre que l'expression « voir... comme... » traduit l'expérience d'un changement sémantique, c'est-à-dire une expérience vécue. Ici, la notion même d'écosystème se voit investie d'une nouvelle signification motivée par son rapprochement avec le système industriel.

Plus précisément, cette expérience vécue est avant tout une expérience imaginative qui permet de saisir une congruence entre le système industriel et l'écosystème. Toutefois, un rapprochement entre ces deux représentations ne conduit pas à les identifier. Il s'agit seulement de percevoir entre elles une ressemblance. Le métabolisme d'un écosystème biologique demeure qualitativement différent, par exemple, de celui d'un système industriel,

\footnotetext{
11 En ce sens, le modèle n'a jamais seulement une fonction descriptive et/ou explicative. Il est aussi censé permettre d'anticiper l'évolution de la situation qu'il redécrit (la prédiction). Et il importe de ne pas confondre cet aspect avec ce que d'aucuns nomment le registre normatif de l'écologie industrielle. Il faut néanmoins aussitôt relativiser cette fonction prédictive. Car, si l'on se place dans la perspective de la théorie des systèmes complexes autoorganisés, une telle prédiction n'est plus vraiment possible (voir la discussion de Ehrenfeld [2007] dont il sera question plus loin).
} 
aussi proche soit-il du fonctionnement de l'écosystème naturel.

Enfin, l'aspect distinctif de la ressemblance métaphorique par rapport à une ressemblance strictement empirique réside dans sa dimension affective. L'expérience de voir le système industriel comme un écosystème ne signifie pas simplement l'attribution de propriétés de l'écosystème à l'activité industrielle. Cette dernière est plutôt un phénomène intériorisé par celui qui « voit... comme... » pour être simultanément extériorisé sur un autre plan, celui de l'écologie. Le sens originel du concept d'écosystème perd de sa pertinence objective, et, sur la base de cette im-pertinence, émerge alors une signification inédite.

Les caractéristiques d'une biocénose - ainsi, par exemple et entre autres, celle qui consiste en des associations bien particulières de populations d'organismes de différentes espèces - ne sauraient plus s'appliquer telles quelles au système industriel. Elles ne désignent plus des propriétés objectives, indépendantes de la perception que l'on peut en avoir. Elles sont désormais rapportées à une expérience particulière du système industriel, à une vision subjective de cet état de choses ${ }^{12}$.

Dès lors que les concepts de l'écologie scientifique servent de métaphores pour l'activité industrielle et économique, il faut être clair sur les conséquences d'une telle opération. Avec la représentation métaphorique, nous avons affaire d'abord à une signification inédite et, par là, largement indéterminée ${ }^{13}$.

En outre, et dans le même ordre d'idées, les métaphores écologiques de l'écologie industrielle n'ont à proprement parler aucune référence propre. Du fait que la signification métaphorique est nouvelle, singulière, on ne peut l'identifier distinctement de manière à pouvoir établir une relation avec un état de choses circonscrit. Contrairement aux apparences, une métaphore comme : «Le système industriel est un écosystème », ne renvoie pas à un référent défini.

Finalement, nous venons de constater que l'emprunt de notions à l'écologie - dans leur emploi métaphorique va de pair avec le fait de mettre entre parenthèses la relation référentielle au sein du domaine d'emprunt. Or, cette impertinence sémantique entraîne logiquement, de surcroît, une discontinuité du même genre, mais au niveau des principes de l'écologie scientifique. L'interconnexion

\footnotetext{
12 Si la métaphore exprime bel et bien une vision subjective des choses, elle ne doit pas forcément se restreindre au point de vue d'une personne. Il peut tout aussi bien s'agir de la perspective d'une communauté de recherche, par exemple. Dans ce cas, on peut parfaitement parler de métaphore collective sans rien modifier aux traits de la métaphore tels que nous les avons décrits précédemment.

${ }^{13}$ Il s'ensuit bien entendu une perturbation d'autres champs sémantiques latents, qu'il s'agisse de celui dont relève l'écologie scientifique ou bien de celui auquel se rattachent les écologistes.
}

ou l'interdépendance, l'intégration dans le tout de la biosphère, la complexité, la coopération, etc., tous ces principes ne sont plus forcément des présupposés de la pensée. Ils perdent en tout cas leur valeur factuelle en tant qu'ils constituent les bases de la science des écosystèmes : il en va ici de la cohérence du savoir ${ }^{14}$.

Un autre type de discours prend désormais le relais. Dans ce discours non plus, les concepts ne sont pas indépendants de celui qui les forge. Un tel langage met en jeu un niveau proprement philosophique que l'épistémologue Gilles-Gaston Granger (1988) qualifie de «méta-conceptue $1^{15}$ ». Il est de surcroit indissociable de l'expérience vécue.

Le développement ci-dessus devrait suffire pour montrer maintenant en quoi ce double usage de l'analogie est bien la source d'une méprise épistémologique.

\section{La méprise épistémologique}

Une grande partie du travail pratique de l'écologie industrielle consiste à établir des bilans de masse et d'énergie (métabolisme industriel) ou à analyser les échanges entre les acteurs d'un ensemble d'activités industrielles (symbiose industrielle). Cette situation correspond clairement à une conception modélisante des notions écologiques. $\mathrm{Et}$, dans ce registre, je ne vois guère de difficulté.

Mais l'écologie industrielle comprend aussi un volet plus théorique qui concerne ses fondements, ses enjeux, son usage de la technologie, ses liens avec d'autres disciplines, etc. Bref, l'écologie industrielle ne se limite pas à des études de symbiose ou de métabolisme industriels. Sur ce plan théorique, au contraire, Ehrenfeld (2007) l'a bien compris : les concepts de l'écologie scientifique ne peuvent pas être réduits à des modèles. Ils expriment la vision singulière $d^{\prime}$ 'un chercheur ou d'une communauté de recherche (cf. Hess, 2003). À moins d'être au clair sur la différence entre modèle et métaphore, nous sommes dès lors inévitablement conduits à des erreurs.

\footnotetext{
${ }^{14}$ La remise en question du caractère référentiel d'un concept comme celui de l'écosystème se répercute aux autres notions de la théorie et a fortiori à ces propositions de base. Il s'agit là d'une application du holisme épistémologique soutenu en son temps par Pierre Duhem dans le domaine de la physique et élargi à l'ensemble de la connaissance par le philosophe W.V. Quine.

${ }^{15}$ Le holisme épistémologique implique au minimum un réexamen des concepts de base de la théorie scientifique en question. Or, un tel réexamen se situe bien à un niveau qui redouble le niveau théorique de départ. Ce redoublement renvoie à une dimension méta-conceptuelle de la réflexion que l'épistémologue G.-G. Granger définit précisément comme celle dont relève la philosophie.
} 


\section{Le piège de l'idéologie}

La première erreur serait de confondre deux usages du discours et de prendre la métaphore pour un modèle. On assisterait alors à un véritable glissement sémantique. Celui-ci permettrait de faire passer un point de vue particulier sur l'activité industrielle pour une propriété objective de cette activité. Cette confusion, l'un des travers de l'idéologie, est d'autant plus pernicieuse qu'elle n'est souvent pas intentionnelle.

Un article de Brad Allenby (1999a), l'un des pionniers de l'écologie industrielle, fournit un bon exemple d'un tel glissement sémantique. Dans cet essai précurseur sur la géoingénierie, l'auteur s'efforce de montrer l'apport de l'écologie industrielle à une ingénierie appliquée au système Terre ${ }^{16}$. La technologie en question comprend, entre autres, la séquestration du gaz carbonique (dioxyde de carbone) dans les profondeurs marines, ou l'injection de dioxyde de soufre dans la stratosphère, etc.

Tout au long de cette réflexion, l'écologie industrielle - et donc les concepts qu'elle a forgés - semble être envisagée naturellement comme une approche intégrative, élargie et multidisciplinaire des systèmes industriels. La suggestion de B. Allenby, si je l'entends bien, consiste à appliquer également cette approche aux systèmes dont s'occupe la géoingénierie.

Par cette assimilation, l'auteur modifie, à première vue sans le savoir, le statut épistémologique des concepts de l'écologie industrielle. Pourquoi ? C'est ce que nous allons tâcher de comprendre. Un modèle, avons-nous dit, diffère de la métaphore au moins sur deux points essentiels. Le modèle a d'abord pour objectif de simplifier les phénomènes; grâce à cette simplification, il élargit le champ d'application de l'écologie scientifique au domaine des activités industrielles et économiques. Ensuite, tout modèle renvoie à un état de choses existant; il réfère à un système d'activités qu'il s'efforce de réorganiser.

Rien de tel n'a lieu dans la géoingénierie. En effet, la notion d'écosystème naturel ne semble pas en mesure de servir de modèle pour les activités incriminées à l'échelle du système Terre. L'activité consistant à agir sur le système Terre présente en effet une difficulté autrement plus grande qu'une activité industrielle clairement délimitée au niveau d'une région ou pour un produit. L'échelle est qualitativement différente. Le problème ici est bien de se représenter la complexité des systèmes de la géoingénierie ${ }^{17}$.

\footnotetext{
${ }^{16}$ La géoingénierie, telle que je l'entends ici, concerne l'application technologique intentionnelle à l'échelle globale du système Terre. Elle ne comprend pas les applications technologiques locales dont les effets (non intentionnels) sont globaux, comme par exemple le réchauffement climatique.

17 Si les modèles classiques simplifient la réalité, les modèles dit « complexes » constituent, eux aussi, une simplification du réel, dans la mesure où leur développement n'est pas maîtrisable.
}

Par ailleurs, les activités de gestion à l'échelle des océans ou de la stratosphère, par exemple, ne sont pas (encore) réelles. Et il pourrait s'avérer que, fussent-elles un jour réalisables, elles ne soient pas désirables pour des raisons éthiques.

Si donc la proposition de B. Allenby a un sens, elle ne saurait être imputée à une interprétation modélisante des concepts de l'écologie industrielle. La distinction entre le modèle et la métaphore offre toutefois une autre solution. En fait, et au vu de ce qui précède, Allenby emploie - volontairement ou non - les notions de l'écologie industrielle non pas comme des modèles, mais bien comme des métaphores. Cet usage métaphorique permet à l'auteur d'exprimer sa représentation personnelle de l'activité technologique ${ }^{18}$. Cette représentation réside d'abord dans une vision de l'intégration de l'homme dans le tout de la nature (la biosphère), où la séparation entre monde naturel et monde humain est perçue comme artificielle (Allenby, 1999a).

En outre, et de façon plus implicite, sa conception de la technologie est celle d'une intervention humaine à outrance dans la nature. Elle tend à « artificialiser » la nature plutôt qu'à «naturaliser » la technologie. Allenby (1999a, p. 83) prône en effet une posture où, écrit-il, «je suis responsable activement pour le monde et pour tout ce qu'il contient, et je peux décider de ce qui vit et de ce qui meurt grâce à une intervention et une gestion actives des systèmes naturels fondamentaux ».

Mais où donc se dissimule l'idéologie, dans ce cas? Selon l'une de ses définitions, l'idéologie définit une situation où, d'un côté, des concepts d'une discipline prétendent à l'objectivité scientifique alors que, de l'autre, on s'en sert dans un contexte où une telle prétention n'a pas lieu d'être. Dans le cas qui nous occupe, elle apparaît quand l'écologie industrielle et ses concepts sont au service d'une vision du monde, comme celle prônée par Allenby. En effet, celui-ci défend clairement l'écologie industrielle comme une approche objective des activités industrielles, y compris dans leurs dimensions économique, sociale et culturelle (Allenby, 1999b). On en déduit par conséquent que, pour lui, les analogies au fondement de l'écologie industrielle relèvent du modèle et non de la métaphore.

Cette imprévisibilité oblige, paradoxalement, à revenir au système réel que le modèle complexe est censé représenter. Le philosophe Jean-Pierre Dupuy (2002) en tire un argument pour soutenir la thèse d'une incertitude de principe. Celle-ci porte en particulier sur les phénomènes environnementaux dont s'occupe la géoingénierie.

18 Dans le contexte d'une délimitation du champ d'études de l'écologie industrielle (cf. Allenby, 1999b), il est significatif de lire chez Frank Boons et Nigel Roome (2001) une critique similaire à l'égard de B. Allenby, avec des conclusions qui vont dans le même sens que les miennes ( $c f$. infra). 
Nous avons toutefois souligné que, pour les systèmes de la géoingénierie, l'échelle descriptive change et avec elle, également, le contexte. Employer les concepts de l'écologie industrielle dans ce nouveau cadre n'implique plus de décrire une propriété objective d'un système. Ces concepts sont repris dans un autre usage - métaphorique que l'on peut juger plus ou moins pertinent en fonction des changements qu'il induit (cf. Hess, 2003). Mais ils dépendent désormais d'un point de vue subjectif. Prétendre le contraire, c'est précisément tomber dans le piège de l'idéologie et commettre un glissement sémantique. Or, à mon sens, c'est ce que fait Allenby quand il revendique l'approche de l'écologie industrielle pour justifier une ingérence et une économie technologiques de l'environnement maximales à l'échelle globale de la Terre.

\section{Un problème de cohérence}

Venons-en maintenant à une seconde erreur. Respecter la différence entre métaphore et modèle est une chose; conserver cette distinction épistémologique jusque dans ses conséquences en est une autre. La confusion repose cette fois sur une omission. Celle-ci consiste à occulter l'incidence d'un emploi métaphorique des concepts de l'écologie industrielle sur le statut a priori factuel des principes écologiques sous-jacents.

En suspendant l'usage ordinaire des notions écologiques pour les appliquer au domaine des activités industrielles et économiques - suspension inévitable dans une métaphore -, nous sommes aussi nécessairement conduits, je le rappelle, à mettre entre parenthèses le bien-fondé des principes de l'écologie scientifique. Cela signifie, autrement dit, qu'ils ont perdu temporairement la légitimité de fait que leur avait octroyée l'écologie scientifique. Ils seront désormais examinés à la lumière d'une perspective d'un autre ordre que scientifique, c'est-à-dire philosophique.

Je désire clarifier ce point en prenant appui sur le travail de J. Ehrenfeld (2007) au sujet du rapport qu'il établit entre l'écologie industrielle et le développement durable. Sa réflexion illustre parfaitement ce que je veux dire. De manière analogue à la mienne, l'auteur prend soin de différencier deux usages des concepts écologiques, à savoir l'analogie et la métaphore (Ehrenfeld, 2003). Et l'emploi respectif qu'il en fait correspond, me semble-t-il, à ce quej'appelle, de mon côté, le modèle et la métaphore ${ }^{19}$. Néanmoins, à lire son texte, on se rend compte qu'il omet de tirer les conséquences - importantes du point de vue épistémologique - associées à cette distinction.

\footnotetext{
${ }_{19}$ Notons cependant qu'Ehrenfeld n'évoque pas explicitement l'aspect heuristique de la métaphore et du modèle. Or, d'une part, cet aspect me paraît essentiel aux investigations de l'écologie industrielle. D'autre part, je crois qu'il est justement à l'origine de l'erreur épistémologique que j'essaie de décrire.
}

Selon lui, le concept classique d'écosystème biologique n'autorise pas un rapprochement entre l'écologie industrielle et le développement durable. C'est pourquoi il propose une idée de l'écosystème qui s'écarte de la conception traditionnelle de l'écologie scientifique. Ce faisant - Ehrenfeld en est parfaitement conscient -, l'écosystème devient une métaphore au sein du discours de l'écologie industrielle : «Ma propre vision normative pour l'écologie industrielle, écrit-il, est fondée sur la métaphore de l'écosystème comme prospère et durable » (Ehrenfeld, 2007, p. 76). Dans cet esprit, il suggère, de façon tout à fait cohérente, de remplacer la théorie écologique classique par une théorie des systèmes émergents auto-organisés (ibid.).

Ce dont l'auteur ne paraît pas avoir conscience, en revanche, c'est que le processus métaphorique dans lequel il s'est engagé implique encore - sous peine de devenir incohérent - l'opacité référentielle des principes sousjacents à la notion examinée. Nous venons de voir en effet que, selon l'exigence de cohérence du savoir scientifique, l'impertinence sémantique suspend de fait la pertinence factuelle de ces principes et présuppose leur examen critique préalable sur le plan philosophique. C'est pourquoi je ne peux manquer de m'interroger : comment Ehrenfeld peut-il, dans le même élan - et quelques lignes à peine après les extraits cités ci-dessus - continuer à s'appuyer sur la légitimité de fait de l'interdépendance comme si, parce qu'elle est un principe de l'écologie classique, elle allait encore de soi ?

En réalité, le problème n'est pas tant l'interdépendance comme telle que sa justification. On rétorquera peut-être qu'il s'agit d'un détail. À vrai dire, ce détail n'en est plus un du point de vue épistémologique, car l'interdépendance dont il s'agit alors n'est plus de l'ordre du fait, mais de la norme. De la (re)description, on est passé à la prescription. Précisons ce point essentiel : la phase dont il est question ici ne doit pas être confondue avec la prédiction permise par le modèle.

Nous l'avons déjà souligné plus haut à propos de l'écosystème biologique conçu comme modèle, la fonction prédictive du modèle s'inscrit bel et bien dans la contingence factuelle des phénomènes. La norme, en revanche, ressortit au registre du devoir-être. Et on ne prescrit pas à partir de la (re)description/prédiction plus ou moins objective d'un état de choses par le modèle ; on prescrit à partir de quelque chose qui n'existe pas indépendamment d'un point de vue, d'une vision du monde et d'un ensemble de valeurs. C'est donc sur ce plan qu'il faudra chercher le bien-fondé de la norme ${ }^{20}$. Après tout, n'est-ce pas ce

${ }^{20}$ Cf. également Korhonen (2004) et Boons et Roome (2001). Mais, contrairement à ce qu'en disent ces auteurs, la démarcation entre modèle et métaphore, telle que j'ai tenté de l'esquisser, montre justement qu'en passant de l'un à l'autre, on renonce à l'objectivité scientifique. Ce passage explicite à un autre type de discours - philosophique - prémunit donc contre le risque d'un 
qu'Ehrenfeld lui-même suggère, par exemple, à propos de la durabilité (Ehrenfeld, 2004 et 2007) ? Ses notations sur l'amour et sur l'espoir d'un monde futur prospère et durable (Ehrenfeld, 2007) invitent à le croire.

\section{Conclusion : écologie industrielle et développement durable}

Je me suis efforcé de montrer que la distinction entre modèle et métaphore est pertinente pour déceler les erreurs épistémologiques que les représentants du champ de l'écologie industrielle peuvent éventuellement commettre. Ils n'y échapperont qu'à la condition d'élucider les usages qu'on y fait de notions empruntées à l'écologie scientifique classique, voire, comme le propose par exemple Ehrenfeld, à des théories du vivant plus contemporaines, mais également plus controversées. À défaut $\mathrm{d}^{\prime}$ une telle clarification, ils risquent fort de commettre des méprises.

L'écologie industrielle se présente régulièrement comme une façon, parmi d'autres, de mettre en œuvre le développement durable. Cette dernière notion est toutefois loin d'être univoque. On s'accorde aujourd'hui pour distinguer une version «faible» d'une version « forte» du développement durable. Dans la première, on le sait, on accepte la substitution du capital naturel par un capital reproductible; cette version se range plutôt du côté de l'économie néoclassique (Solow, 1997). La seconde trouve au contraire ses partisans surtout parmi les écologues, persuadés par l'idée que le capital naturel est irremplaçable (Daly, 1997).

Je pense, pour ma part, que, sans l'apport de distinctions philosophiques - $\mathrm{d}$ 'ordre épistémologique en particulier -, les tenants de l'écologie industrielle entretiennent finalement la divergence sémantique qui grève le concept de développement durable. Ne vont-ils pas ainsi à l'encontre de leur idéal? Toujours est-il qu'en s'appuyant sur la pensée philosophique, ils disposent de concepts et de ressources argumentatives pour soutenir une version «forte» du développement durable. Car une telle version repose sur des principes écologiques non plus factuels mais normatifs, c'est-à-dire ceux-là mêmes que la philosophie a finalement pour tâche de justifier aussi au sein de l'écologie industrielle ${ }^{21}$.

paralogisme naturaliste, permettant ainsi une justification de la norme.

${ }^{21}$ Je remercie les professeurs Dominique Bourg et Suren Erkman, de l'Université de Lausanne, pour leurs remarques formulées lors des versions antérieures de ce travail, Théodore Besson, Frédéric Piguet et un rapporteur anonyme de la revue pour leurs suggestions bibliographiques, ainsi que Muriel Gilbert pour sa relecture minutieuse du manuscrit.

\section{Références}

Allenby, B., 1999a. Earth systems engineering: The role of industrial ecology in an engineered world, Journal of Industrial Ecology, 2, 3, 73-93.

Allenby, B., 1999b. Culture and industrial ecology, Journal of Industrial Ecology, 3, 1, 2-4.

Aristote, 1991. Rhétorique, trad. C.E. Ruelle, revue par P. Vanhemerlyck, Paris, Le Livre de Poche.

Bey, C., 2001. Quo vadis industrial ecology? Realigning the discipline with its roots, Greener Management International, 34, 35-42.

Black, M., 1962. Models and Metaphors, Ithica, Cornell University Press.

Black, M., 1979. More about Metaphor, in Ortony, A. (Ed.), Metaphor and Thought, Cambridge, Cambridge University Press, 19-43.

Booms, F., Roome, N., 2001. Industrial ecology as a cultural phenomenon. On objectivity as a normative position, Journal of Industrial Ecology, 4, 2, 49-54.

Daly, H.E., 1997. Reply: Georgescu-Roegen versus SolowStiglitz, Ecological Economics, 22, 3, 261-266.

Dupuy, J.-P., 2002. Pour un catastrophismeéclairé: quand l'impossible est certain, Paris, Le Seuil.

Ehrenfeld, J.R., 2003. Putting a spotlight on metaphors and analogies in industrial ecology, Journal of Industrial Ecology, $7,1,1-4$.

Ehrenfeld, J.R., 2004. Can industrial ecology be the "science of sustainability"?, Journal of Industrial Ecology, 8, 1-2, 1-3.

Ehrenfeld, J.R., 2007. Would industrial ecology exist without sustainability in the background?, Journal of Industrial Ecology, $11,1,73-84$.

Erkman, S., 2004. Vers une écologie industrielle : comment mettre en pratique le développement durable dans une société hyperindustrielle, Paris, Charles Léopold Mayer.

Granger, G.G., 1988. Pour la connaissance philosophique, Paris, Odile Jacob.

Hess, G., 2003. Métaphore, science, philosophie, Revue de théologie et de philosophie, 135, 115-135.

Hess, G., 2004. L'innovation métaphorique et la référence selon Paul Ricœur et Max Black : une antinomie philosophique, Revue philosophique de Louvain, 102, 630-659.

Isenmann, R., 2003. Further efforts to clarify industrial ecology's hidden philosophy of nature, Journal of Industrial Ecology, 6 , 3-4, 27-48. (Une version abrégée de ce texte existe sous le titre suivant : Industrial ecology: Shedding more light on its perspective of understanding nature as model, Sustainable Development, 11 [2003], 143-158.)

Korhonen, J., 2004. Theory of industrial ecology, Progress in Industrial Ecology: An International Journal, 1, 1/2/3, 61-85.

Korhonen, J., 2005. Do we really need the debate on the natural ecosystem metaphor in technology management and sustainable development literature ?, Clean Technical Environmental Policy, 7, 33-41.

Lakoff, G., Johnson, M., 1980. La Métaphore dans la vie quotidienne, Paris, Minuit.

Levine, S.H., 2003. Comparing products and production in ecological and industrial systems, Journal of Industrial Ecology, 7, 2, 33-42.

Nouvel, P., 2000. L'Art d'aimer la science, Paris, PUF.

Ricœur, P., 1975. La Métaphore vive, Paris, Le Seuil.

Solow, R.M., 1997. Reply: Georgescu-Roegen versus Solow/Stiglitz, Ecological Economics, 22, 267-268. 
Varenne F., 2006. Les Notions de métaphore et d'analogie dans les épistémologies des modèles et des simulations, Paris, Pétra.

Wells, P.E., 2006. Re-writing the ecological metaphor. Part 1 , Progress in Industrial Ecology: An International Journal, 3, 1/2, 114-128.
Wells, P.E., Darby, L., 2006. Re-writing the ecological metaphor Part 2: The example of diversity, Progress in Industrial Ecology: An International Journal, 3, 1/2, 129-147.

Wittgenstein, L., 1953. Investigations philosophiques, Paris, Gallimard. 